\title{
Glioblastoma Following Ischemic Stroke
}

\author{
Naif M. Alotaibi, Nardin Samuel, Alireza Mansouri, Romina Nejad, \\ R. Loch Macdonald
}

Keywords: Glioblastoma, Ischemic brain injury, Stroke

doi:10.1017/cjn.2017.220

Can J Neurol Sci. 2017; 44: 732-733

A 59-year-old man presented with a progressive decline in speech. Past medical history was significant for a left middle cerebral artery (MCA) territory stroke 8 years prior to the current presentation (Figure 1A,B,C). Neurologic examination demonstrated global aphasia and preexisting right-sided hemiplegia. Imaging showed a large enhancing lesion within the infarcted area
(Figure 1D,E,F). Computed tomography (CT) of the chest, abdomen, and pelvis did not reveal any evidence of other tumours. A presumed diagnosis of a primary neoplastic lesion was made, and the patient underwent craniotomy and subtotal resection of the lesion. There were no intra- or postoperative complications. Final histopathology was consistent with a WHO grade IV
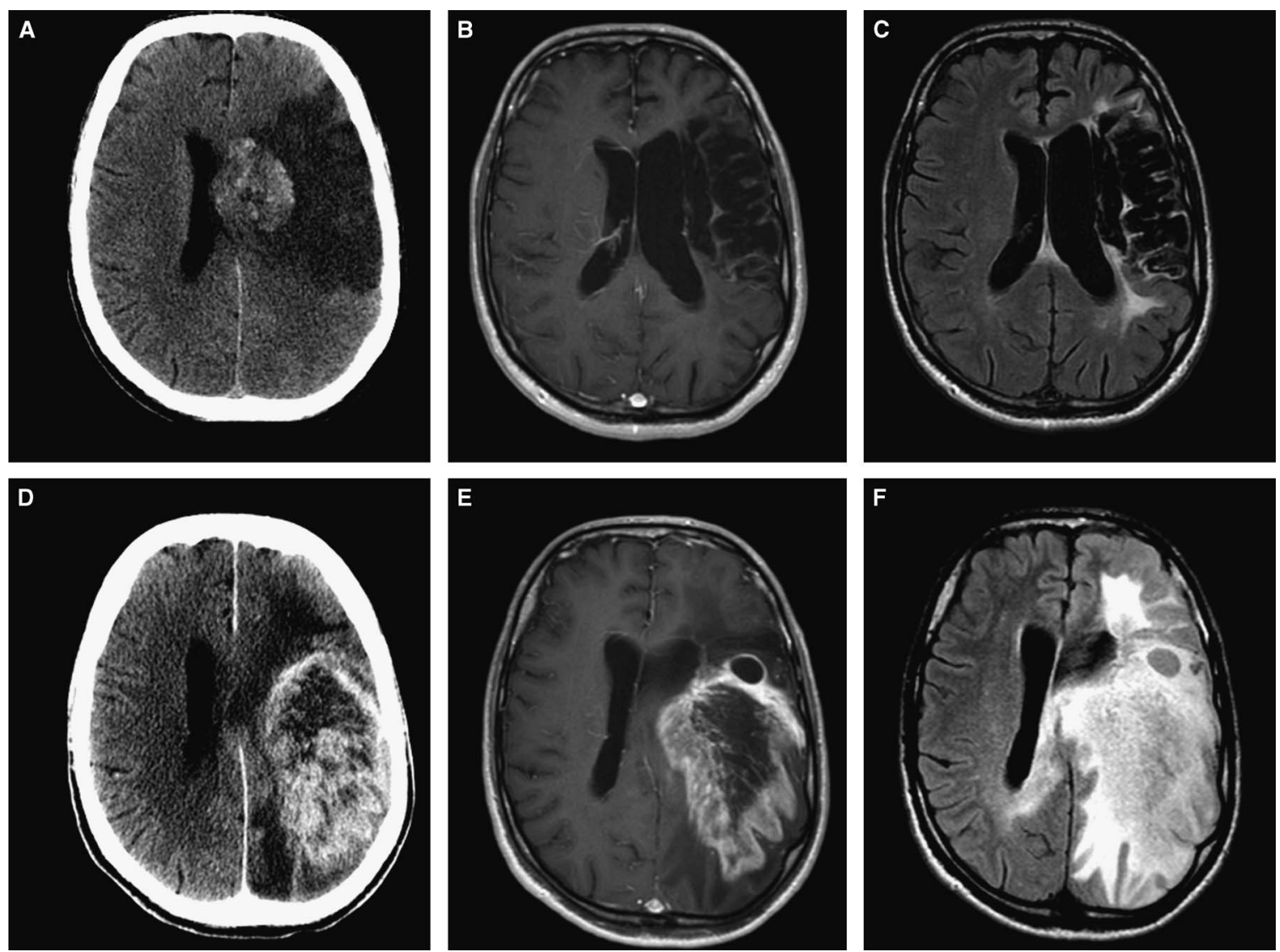

Figure 1: Initial neuroimaging at the time of stroke $(A, B, C)$ : axial $C T(A)$, axial MRI with gadolinium (B), and FLAIR sequence $(C)$. Eight years later $(D, E, F)$ : axial $C T(A)$, axial MRI with gadolinium $(B)$, and FLAIR sequence $(C)$ showing large intra-axial tumour involving the same area affected by ischemia.

From the Department of Surgery, Division of Neurosurgery, University of Toronto, Toronto, Ontario, Canada (NMA, NS, AM, RN, RLM); Division of Neurosurgery, St. Michael's Hospital, Labatt Family Centre of Excellence in Brain Injury and Trauma Research, University of Toronto, Ontario, Canada (RLM).

Received September 3, 2016. Final Revisions Submitted April 13, 2017. Date of Acceptance May 27, 2017.

Correspondence to: Naif M. Alotaibi, Department of Surgery, Division of Neurosurgery, University of Toronto, 399 Bathurst Street, Toronto, Ontario, Canada WW 4-427.

Email: naif.alotaibi@mail.utoronto.ca. 
glioblastoma. The patient was referred for radiation therapy. His Karnofsky score at 1-month follow-up was $40 \%$.

Cases of de-novo glioblastoma have been reported following other intracranial insult or pathology, including penetrating head injury, cerebral contusion, and previous surgical resection. ${ }^{1}$ Possible aetiologies that have been proposed include loss of integrity of the blood-brain barrier, the presence of free radicals, and loss of immune surveillance. ${ }^{2}$ Although there are cases and studies demonstrating the occurrence of infarctions secondary to glioblastoma, ${ }^{3-5}$ reports of the development of glioblastoma in regions of previous cerebral infarction are exceedingly rare. ${ }^{6,7}$ Wojtasiewicz et al. ${ }^{7}$ reported the first pathologically proven case of de-novo glioblastoma arising from within a large infracted tissue after 2 years from the time of MCA stroke in a 73-year-old woman. To the best of our knowledge, our case is the only one demonstrating glioblastoma development in an ischemic zone later than 8 years after the time of the ischemic insult.

The present case raises the question of how ischemia and gliosis may play a role in the tumorigenesis of de-novo glioblastoma. ${ }^{6}$ It has been posited that brain tissue repair mechanisms mirror tumorigenesis and that reactive gliosis may lead to an increased risk of accumulating deleterious mutations. ${ }^{8}$ Similarly, the relationship between glioblastoma and a hypoxic microenvironment is well-documented, and glioblastomas are distinguished histopathologically from lower-grade infiltrative astrocytomas by characteristic necrotic foci in ischemic zones. ${ }^{9} \mathrm{Kim}$ et al. ${ }^{10}$ recently identified key metabolic pathways that drive survival of these malignant cells in zones of hypoxia. They found high expression of mitochondrial serine hydroxymethyltransferase (SHMT2) in human glioblastoma, which allows cancer cells to reduce their oxygen needs and to be in a metabolic state characterized by prolonged survival in the avascular zones of the tumour.

Taken together, this report raises questions about an important area of glioblastoma biology, demonstrating that this malignant tumour may have a predilection for hypoxic zones of the brain in patients who have suffered ischemic stroke. Future studies aimed at systematically characterizing the epidemiological incidence of glioblastoma post-infarction may be warranted in order to better gauge the clinical impact of this molecularly significant relationship.

\section{DisCLOSURES}

Naif Alotaibi, Nardin Samuel, Alireza Mansouri, Romina Nejad, and R. Loch Macdonald hereby declare that they have no conflicts of interest to disclose.

This manuscript has not been previously published and is not under consideration in the same or a substantially similar form in any other peer-reviewed media. All authors listed have contributed sufficiently to the project to be included as authors, and all those who are qualified to be authors are listed in the author by-line.

\section{REFERENCES}

1. Yaghmour W, Kurdi ME, Baeesa SS. De novo glioblastoma in the territory of a recent middle cerebral artery infarction and a residual meningioma: pathogenesis revisited. World J Surg Oncol. 2016; $14: 112$.

2. Kaur B, Khwaja FW, Severson EA, Matheny SL, Brat DJ, Van Meir EG. Hypoxia and the hypoxia-inducible-factor pathway in glioma growth and angiogenesis. Neuro Oncol. 2005;7(2):134-53.

3. Rojas-Marcos I, Martin-Duverneuil N, Laigle-Donadey F, Taillibert $\mathrm{S}$, Delattre JY. Ischemic stroke in patients with glioblastoma multiforme. J Neurol. 2005;252(4):488-9.

4. Pina S, Carneiro A, Rodrigues T, et al. Acute ischemic stroke secondary to glioblastoma: a case report. Neuroradiol J. 2014; 27(1):85-90.

5. Kamiya-Matsuoka C, Cachia D, Yust-Katz S, et al. Ischemic stroke in patients with gliomas at The University of Texas-M.D. Anderson Cancer Center. J Neurooncol. 2015;125(1):143-8.

6. Ojemakinde $\mathrm{O}$, Gonzalez Toledo E, Wilson J. A case of glioblastoma in infarcted brain. J La State Med Soc. 2013;165(1):30-2.

7. Wojtasiewicz TJ, Ducruet AF, Noticewala SS, Canoll P, McKhann GM 2nd. De novo glioblastoma in the territory of a prior middle cerebral artery infarct. Case Rep Neurol Med. 2013;2013:356526.

8. Halliday JJ, Holland EC. Connective tissue growth factor and the parallels between brain injury and brain tumors. J Natl Cancer Inst. 2011;103(15):1141-3.

9. Brat DJ, Castellano-Sanchez AA, Hunter SB, et al. Pseudopalisades in glioblastoma are hypoxic, express extracellular matrix proteases, and are formed by an actively migrating cell population. Cancer Res. 2004;64(3):920-7.

10. Kim D, Fiske BP, Birsoy K, et al. SHMT2 drives glioma cell survival in ischaemia but imposes a dependence on glycine clearance. Nature. 2015;520(7547):363-7. 